\title{
Tunneling Measurements of Superconducting Quasi-Particle Density of States and Calculation of Phonon Spectra*
}

\author{
J. M. Rowell \\ Bell Telephone Laboratories, Inc., Murray Hill, New Jersey \\ (October 10, 1969)

\begin{abstract}
It is an unfortunate fact that the tunneling technique, which has proved incredibly successful in the study of superconductivity, has given little information about the normal state properties of metals and semiconductors. It will be shown that, in the determination of the superconducting quasi-particle density of states, it is the change in density induced by the onset of superconductivity which is measured rather than the total density.

Returning to the problem of normal materials, a review of the limited achievements and failures of tunneling will be presented. This will include the influence of band edges on tunneling in $p$ - $n$ diodes and metal-semiconductor contacts, the structures observed in tunneling into bismuth and the negative results obtained in nickel and palladium. The dominant effect 'f the change in barrier shape in most of these tunneling characteristics will be illustrated.
\end{abstract}

Key words: Density of states; phonons; semiconductors; superconductivity; tunneling.

Many of the talks heard this week have outlined experimental techniques which determine the density of electron states in metals and semiconductors over energy ranges which are typically $1-10$ volts. I would like to discuss a technique which is much happier in the range of 1-10 millivolts, is the only method which can measure the change in density of electron states induced by superconductivity, but which to date must be classed as a failure in the determination of band properties of normal metals. For semiconductors and semimetals, because of smaller energies and larger fractional changes in electron density at band edges, the situation is a little better. Even in these materials, however, we can only say that density-of-states effects are observed and cannot generally deduce a density measurement from the experimental results.

After those opening remarks, it will be obvious that most of you attending this conference should not be familiar with the tunneling technique so I will briefly

\footnotetext{
*An invited paper presented at the 3d Materials Research Symposium, Electronic Density of States, November 3-6, 1969, Gaithersburg, Md.
}

review the experimental method. Two structures are commonly used, the metal-insulator-metal (M-I-M) junction, which relies on the oxide of the first metal to form the insulator, and the metal-semiconductor (M-S) contact which uses the depletion layer (Schottky barrier) at the surface of the semiconductor. In the M-I-M case it is generally hard to prepare sufficiently smooth clean surfaces of bulk metal that oxidize in a controlled way so films have been used in all but a few cases. After evaporation of the first metal film ( $\mathrm{Al}, \mathrm{Pb}, \mathrm{Sn}, \mathrm{Mg}$, for example), oxidation takes place by exposure to air, oxygen, or a glow discharge in oxygen, and the second film is then cross evaporated to complete the junction. Typical oxide thicknesses are 15-20 ̊. To make the M-S contact a semiconductor is cleaved in vacuum and covered with the metal very rapidly. The barrier is lower and $\sim 100 \AA$ thick. In some materials, where a suitable Schottky barrier does not form at the surface (e.g. InAs), a M-I-S structure can be made by oxidizing the semiconductor before evaporation of the metal.

These three tunneling structures are shown in figure 1, with the circuit used to measure the current-voltage characteristic or, if greater detail is required, the 
dynamic resistance $(d V) /(d I)$ versus voltage. The figure also shows schematically the potential barrier (with typical energies indicated) which separates the electrodes.

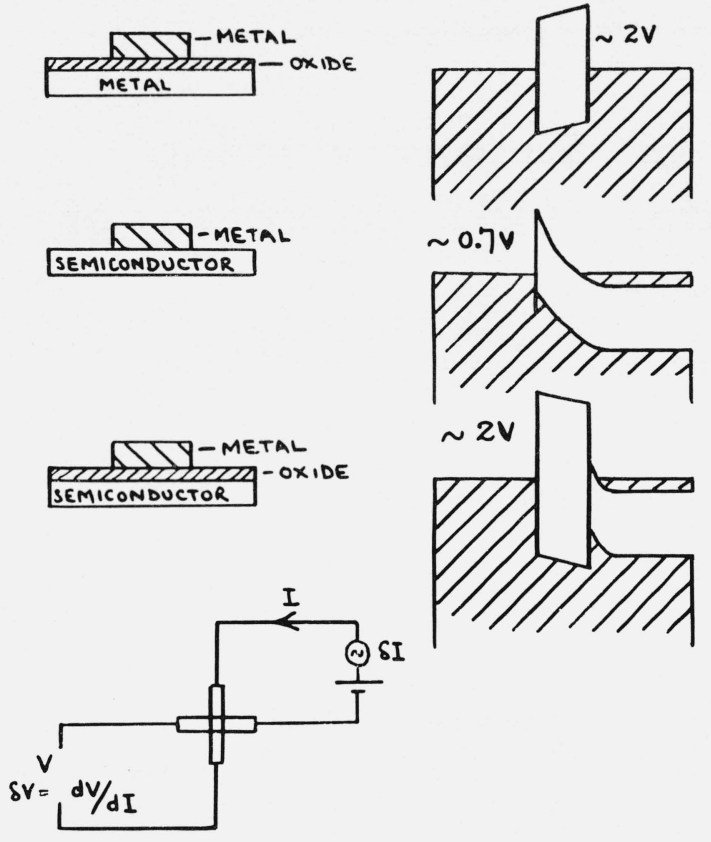

FIGURE 1. Schematics of three widely studied tunneling structures and the circuit used to measure their I-V characteristics.

Considering the M-I-M junction as the most favorable case for studying metals, I would like to make a number of points regarding the theoretical possibilities of observing band structure effects and the probable experimental difficulties.

The tunneling current through a barrier of the type shown in figure 2 is given by [1],

$$
\begin{array}{r}
j=\frac{4 \pi e}{\hbar} \sum_{\mathbf{k}_{t}} \int_{-\infty}^{\infty} d E_{x} \rho_{a}(E) \rho_{b}(E) P\left(E_{x}\right)[f(E) \\
-f(E-e V)],
\end{array}
$$

where $\rho_{a}(E)$ and $\rho_{b}(E)$ are the density of states for a given transverse momentum $k_{t}$ and total energy $E, f(E)$

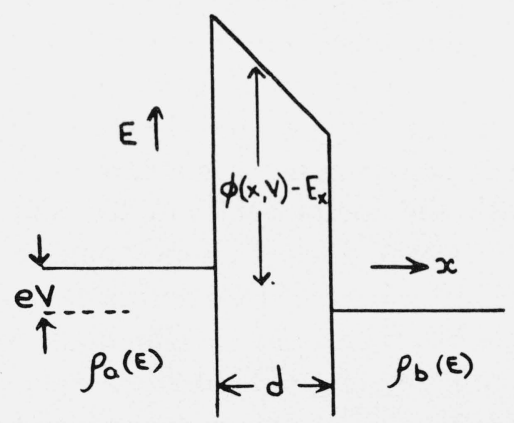

FIGURE 2. The potential in an ideal trapezoidal barrier between metal electrodes. is the usual Fermi distribution and $\boldsymbol{E}_{x}$ the total electron energy perpendicular to the barrier. The tunneling probability $P\left(E_{x}\right)$ has the form

$$
P\left(E_{x}\right)=A \exp \left\{-\frac{2}{\hbar} \int_{0}^{d}\left[2 m\left(\phi(x, V)-E_{x}\right)\right]^{1 / 2} d x\right\}
$$

where $d$ is the barrier thickness and $\left[\phi(x, V)-E_{x}\right]$ is the barrier potential at position $x$ when a voltage $V$ is applied. The pre-exponential factor $A$ describes the frequency with which an electron arrives at the barrier interface and its exact form determines whether one expects to observe density of states effects at all. In the W.K.B. approximation, which makes the metal-barrier interface properties vary slowly compared to the electron wavelength [1], $A$ is proportional to $I /\left[\rho_{a}(E) \rho_{b}(E)\right]$ so that the current in (1) is independent of electron density of states. The other extreme limit, with the metal-barrier interface absolutely sharp, gives a complicated prefactor $A$ which does not exactly cancel the density terms in eq (1) $[1,2]$ so some density of states effect in tunneling might be expected. However, as the interface properties can never be known in this detail, a serious interpretation of any such observation would be impossible. Rather than looking for results of the slow energy variation of $\rho(E)$ within a band, a more likely experiment is to look for effects of band edges, where the number of final available states for the tunneling electron changes more abruptly. It is generally felt that band edges can be observed in tunneling as long as an appreciable fractional change in total electron density is produced by the new band. As we will see later, this usually happens at convenient energies only in semiconductors. Duke [3] has also pointed out that an increased tunneling probability into the new band will enhance the magnitude of the current onset near the band edge.

Apart from the theoretical question of the exact nature of the metal-insulator interface and its role in producing density of states effects, there are a number of serious experimental problems which lead me to question whether these effects could be observed in metals. These problems can be illustrated by reference to figure 3. First, typical barrier heights in oxides are $\sim 2$ volts, which is a small energy compared to interesting band structure in most metals. For an applied bias $>\phi_{R}$, electrons from the Fermi level (at A for example) tunnel into the conduction band of the insulator rather than into the second electrode. Second, the tunneling probability is much greater for electrons at B than for those at $\mathrm{C}$, which enter the second metal just above the Fermi level. In figure $3 \mathrm{~b}$, for example, where 
we assume $\phi_{R}=\phi_{L}=2 \mathrm{~V}$, the transmission probability at $\mathrm{B}$ is $\sim 10^{-9}$, whereas at $\mathrm{C}$ it is $\sim 10^{-16}$. Thus the chance of probing band edges far below the Fermi level in the left metal seems remote, as practically none of the

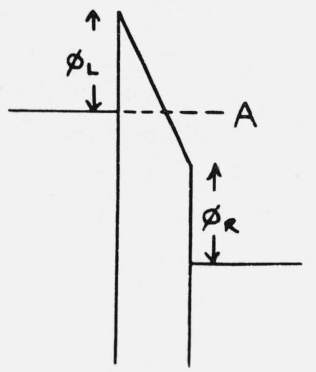

(a)

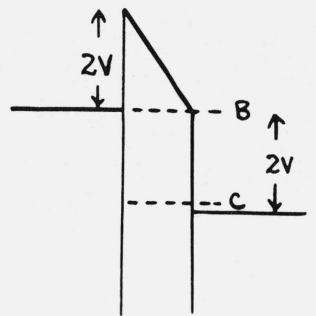

(b)
FigURE 3. Figure (a) shows the possible injection of electrons into the conduction band of the insulator (Fowler Nordheim tunneling).

Figure (b) shows two possible tunneling paths into the second electrode.

current, flows from these levels. The possibility of probing band edges above the Fermi level (at B in the right metal for example) raises the third problem, namely that the current-voltage characteristic of the junction is dominated very strongly by the changes in barrier shape. Typical results obtained by Fisher and Giaever [4] are shown in figure 4. At low voltages the junctions are ohmic (small deviations are observable only in detailed conductance plots) but from 0.2 to $1.4 \mathrm{~V}$ the current depends almost exponentially on voltage. This strong current dependence is purely the result of the barrier becoming lower (for electrons leaving the Fermi level) as the voltage increases. Thus any small changes in current (or conductance) due to band edges, superimposed on this exponential behavior, will be extremely hard to observe. There are also other difficulties which one has to consider in many tunneling experiments. If evaporated metal films are used it is unusual for these to have the properties of single crystals or even clean polycrystals. Even more critical are the surface properties of the films, as tunneling between normal metals is sensitive to the material only within a screening length of the surface. As this surface is in contact with (or diffused into) the insulating oxide the chance of it having bulk properties seems remote.

After this very pessimistic survey of why tunneling is not the right technique for the study of density of states effect in metals, let me review a number of experiments where band edges at least are observed, or affect the tunneling characteristic noticeably.

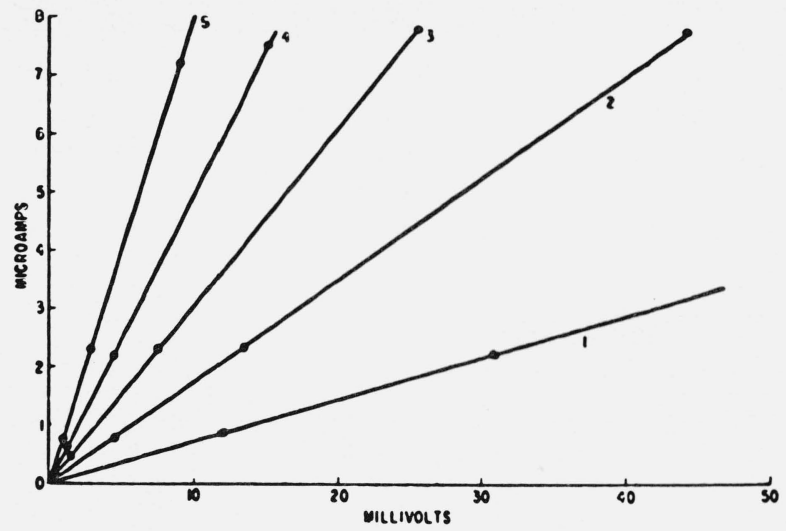

(a)

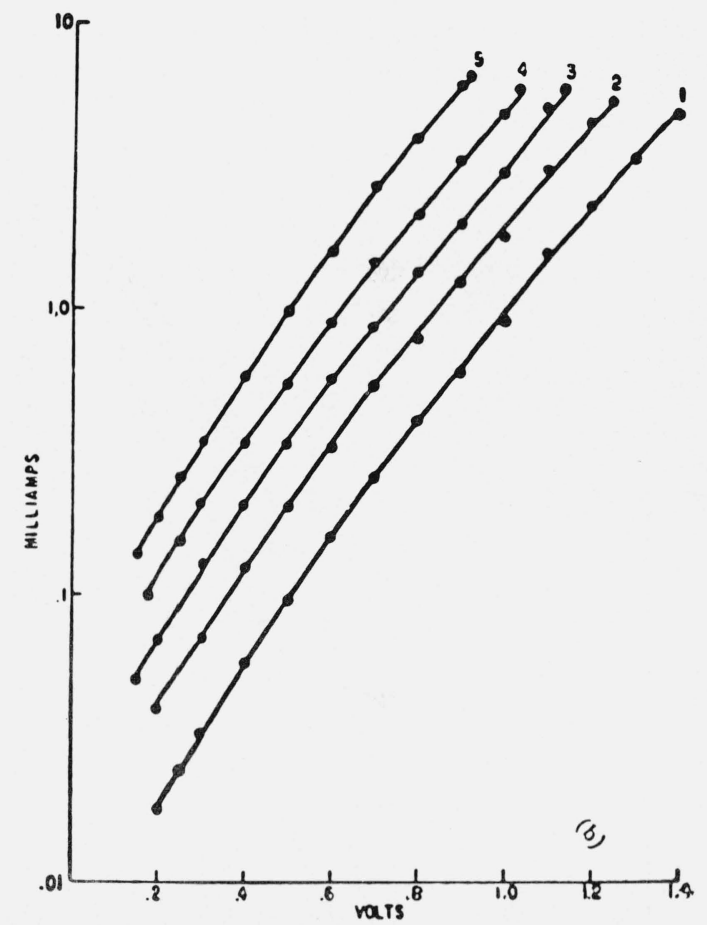

FIGURE 4. I-V characteristics reported by Fisher and Giaever [4]. (a) At low voltages, the current through thin oxide films is proportional to voltage and to film area. Curves shown are for five films with areas in the proportions 5:4:3:2:1 as indicated. (b) At higher voltages, the current increases exponentially with voltage.

\section{Metal-Semiconductor Contacts}

In a heavily doped $n$-type semiconductor the Fermi energy is relatively small $(<100 \mathrm{mV})$ and the depletion layer formed at the surface is thin enough to permit tunneling. Unfortunately the barrier height is rather low and barrier thickness varies with bias, leading to a strong dependence of conductance on voltage. Nevertheless, the calculation of Conley et al. [5] predicts that the minimum conductance occurs when the bottom of the band crosses the Fermi level of the metal (fig. 5). This has been observed in the experiments of 


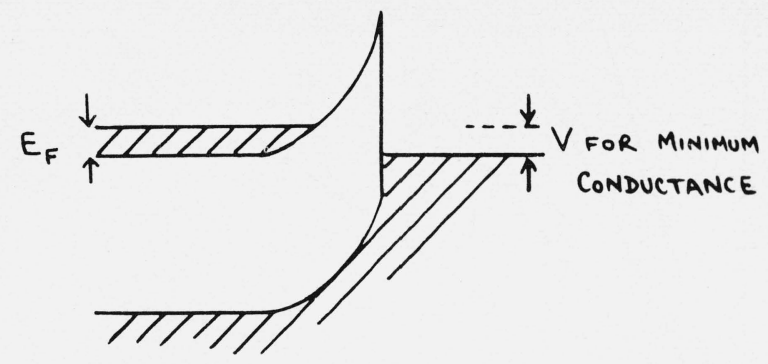

Figure 5. Metal-semiconductor contact with bais applied to produce a minimum in conductance.

Steinrisser et al. [6], as shown in figure 6. The barrier parameters were determined independently and the agreement between calculated and experimental conductances is orders of magnitude better than in most



FIGURE 6. Calculation and measurement of conductance in M-S contacts by Steinrisser et al. [6]. Comparison between three experimentally measured conductance curves on $\mathrm{n}=7.5 \times 10^{18} / \mathrm{cm}^{3} \mathrm{Sb}$ doped Ge [solid lines $(a),(c)$, and $(d)]$ at $4.2 \mathrm{~K}$ and the calculated conductance $\left[\right.$ dashed line (b)] for a barrier height $\mathrm{V}_{b}=0.63 \mathrm{eV}$ obtained from capacitance measurements. The most commonly observed conductance curves were similar to (c), whereas (a) and (d) represent the high-and the low-conductance extremes. The contact metal is $\mathrm{Pb}$ and the contact area is $2.5 \pm 0.5 \times 10^{-4} \mathrm{~cm}^{2}$. Structure associated with the superconducting energy gap has been omitted. The Fermi degeneracy $\mu_{F}=25 \mathrm{mV}$ has been indicated. tunneling systems. The density of states in the semiconductor band was assumed constant in this calculation. The general aim of this type of experiment has been to obtain such a satisfactory agreement with the calculated conductance rather than to determine a density of states variation. Uncertainty in the energy dependence of the barrier parameters would make such a determination exceedingly difficult.

A second band structure effect, observed in Au-Ge surface barrier contacts by Conley and Tieman [7], is the onset of tunneling into the $\mathrm{k}=0$ conduction band minimum, roughly $150 \mathrm{mV}$ above the Fermi level. The influence of the band edge is rather dramatic in this case, as shown in figure 7 , and a decrease of resistance by at least a factor of 10 is observed within $\sim 10 \mathrm{mV}$ of the edge. Note that the band edge is marked by a decrease in resistance, or increase in conductance.

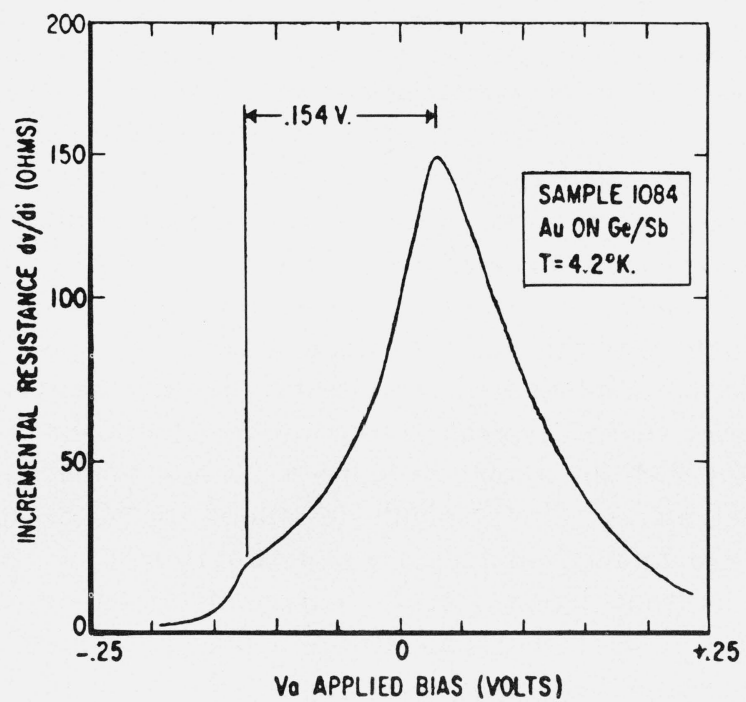

FIGURE 7. Tunneling resistance of a Au-Ge surface barrier contact, measured by Conley and Tiemann [7]. The onset of transitions to the zone-centered $\left(\Gamma_{2}{ }^{\prime}\right)$ conduction band in Ge observed in the incremental resistance $\mathrm{dv} / \mathrm{di}$ at an applied bias $\mathrm{V}_{a}=-0.124 \mathrm{~V}$. Note that that threshold is $0.154 \mathrm{~V}$ from the maximum, a value which corresponds to the interband $\mathrm{L}-\Gamma_{2}{ }^{\prime}$ separation.

\section{Metal-Insulator-Semiconductor Junctions}

In order to investigate tunneling into semiconductors to higher voltages, or study those materials which do not form surface barrier contacts, an oxide can be grown on the surface before evaporation of the metal. Alternatively, for semiconductors which can be evaporated, junctions of the type aluminum-aluminum oxide-semiconductor have been fabricated. The current calculated by Chang et al. [8] for such a structure, on a degenerate $p$-type semiconductor, is shown in figure 8. The current is a maximum at the top of the valence 
band and a minimum at the bottom of the conduction band. At higher voltages it is interesting to see that properties of the insulator, namely the increased tunneling probability at voltages corresponding to the heights $\phi_{R}$ and $\phi_{L}$, dominate the characteristic. Experiments on a number of different III-V and II-VI semiconductors have confirmed this type of behavior except that the

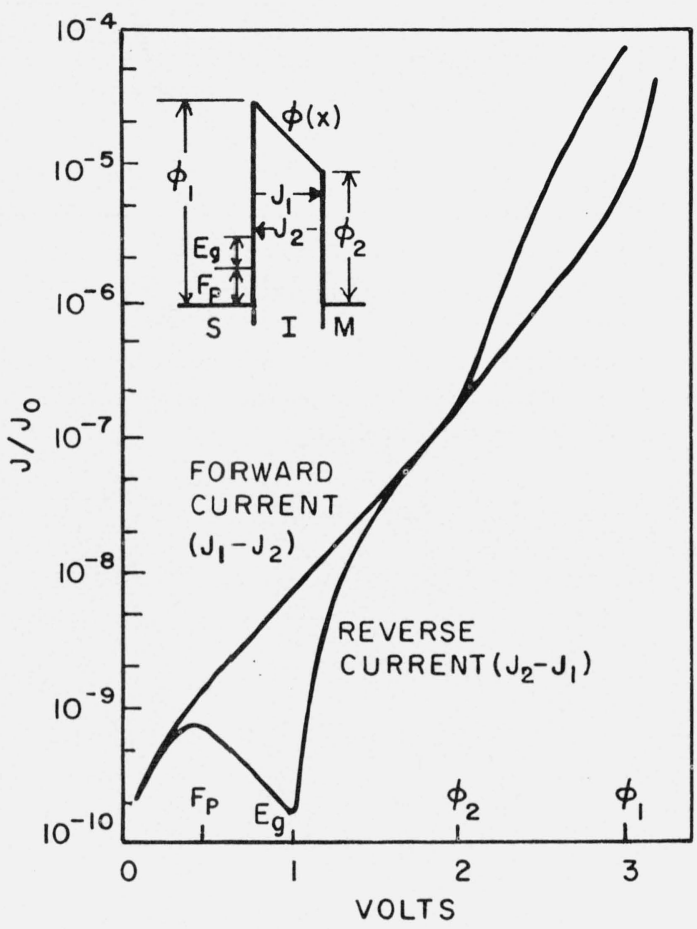

current minimum is not so sharp as the calculated one, as shown in figure 8 for SnTe.

The observation of interface states and impurity bands has also been reported from tunneling studies. The left-hand plots of figure 9 show the conductance results, obtained by Gray [9], for two M-I-S structures with different boron doping levels in silicon.

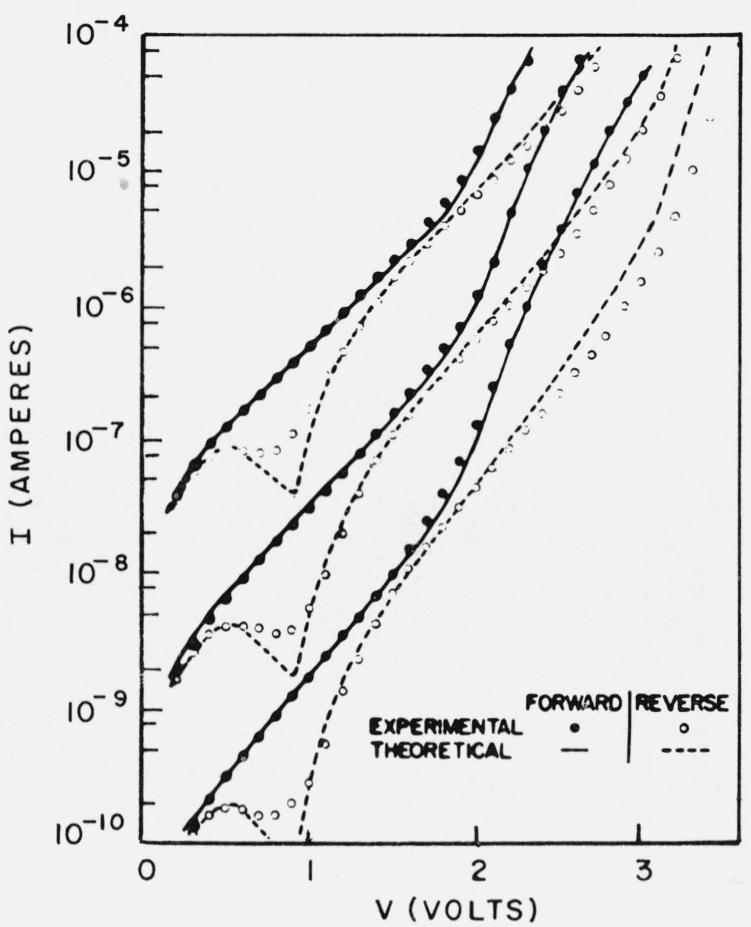

FIGURE 8. Calculation and measurement of current in metal-insulator-semiconductor junctions by Chang et al. [8]. (a) Theoretical current-voltage characteristics for a M-I-S junction. The semiconductor is degenerate $\mathrm{p}$ type and the conduction band of the insulator provides the tunneling barrier. (b) Current-voltage characteristics for Al-Al $\mathrm{O}_{3}$-SnTe junctions at $4.2 \mathrm{~K}$. Three sets of curves are shown corresponding to samples with various oxide thickness.

\section{3. p-n Diodes}

The I-V characteristic of the Esaki diode [10] is a clear observation of the influence of band edges on tunneling but recently this system has received little attention, probably because the barrier profile is difficult to measure. It is also difficult in this case to decide how much of the junction current is due to tunneling.

An interesting effect which has been observed in p-n diodes is the influence of Landau levels on the tunneling conductance. This was first reported by Chynoweth et al. [11] for InSb diodes and extensive studies of $\mathrm{Ge}$ diodes have been made by Bernard et al. [12]. Recently Landau levels in InAs have been measured by Tsui [13] in an M-I-S structure. Because only one electrode is a semiconductor the latter results are simpler to interpret.

\section{Metal-Insulator-Metal Junctions}

This type of tunneling structure is usually comprised of aluminum-aluminum oxide-second electrode of semimetal or metal. Considerable effort by various groups has gone into trying to observe the band edges in bismuth. Tunneling structures at low energies $(<50$ $\mathrm{mV}$ ) have been reported by Esaki et al. [14] but these results have not been reproduced elsewhere [15]. At higher voltages the characteristics for polycrystalline films were first reported by Hauser and Testardi [16]. In figure 10 we show the good agreement between their results and those of Sawatari and Arai [17]. However, the exact position of the bands which give rise to this structure is difficult to determine from the tunneling characteristic. 

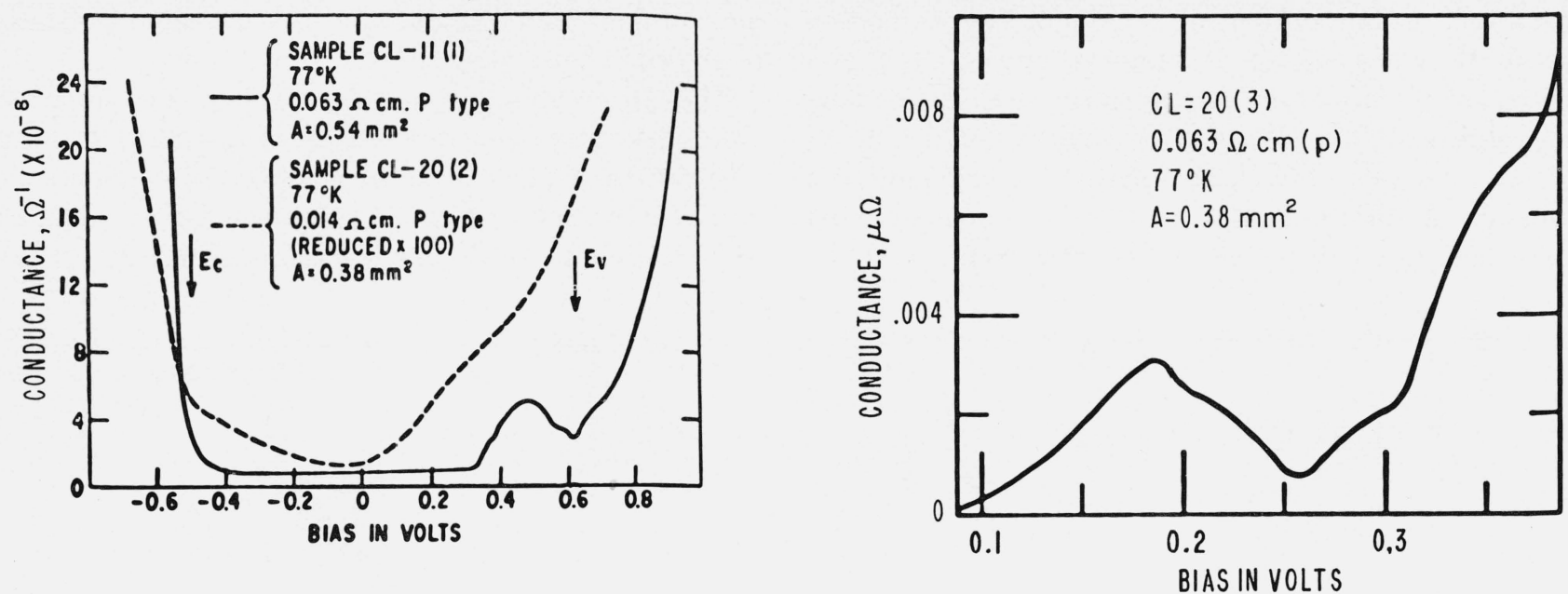

FigURE 9. Observation of impurity levels in tunneling by Gray [9]. (a) de conductance versus bias showing the effect of a boron-impurity band at two different doping levels. The conductance of the more heavily doped sample is reduced $\times 100$ for comparison. (b) Detailed de conductance near an impurity band $(0.19 \mathrm{~V})$. This sample was cleaved in air before mounting in the vacuum jar.
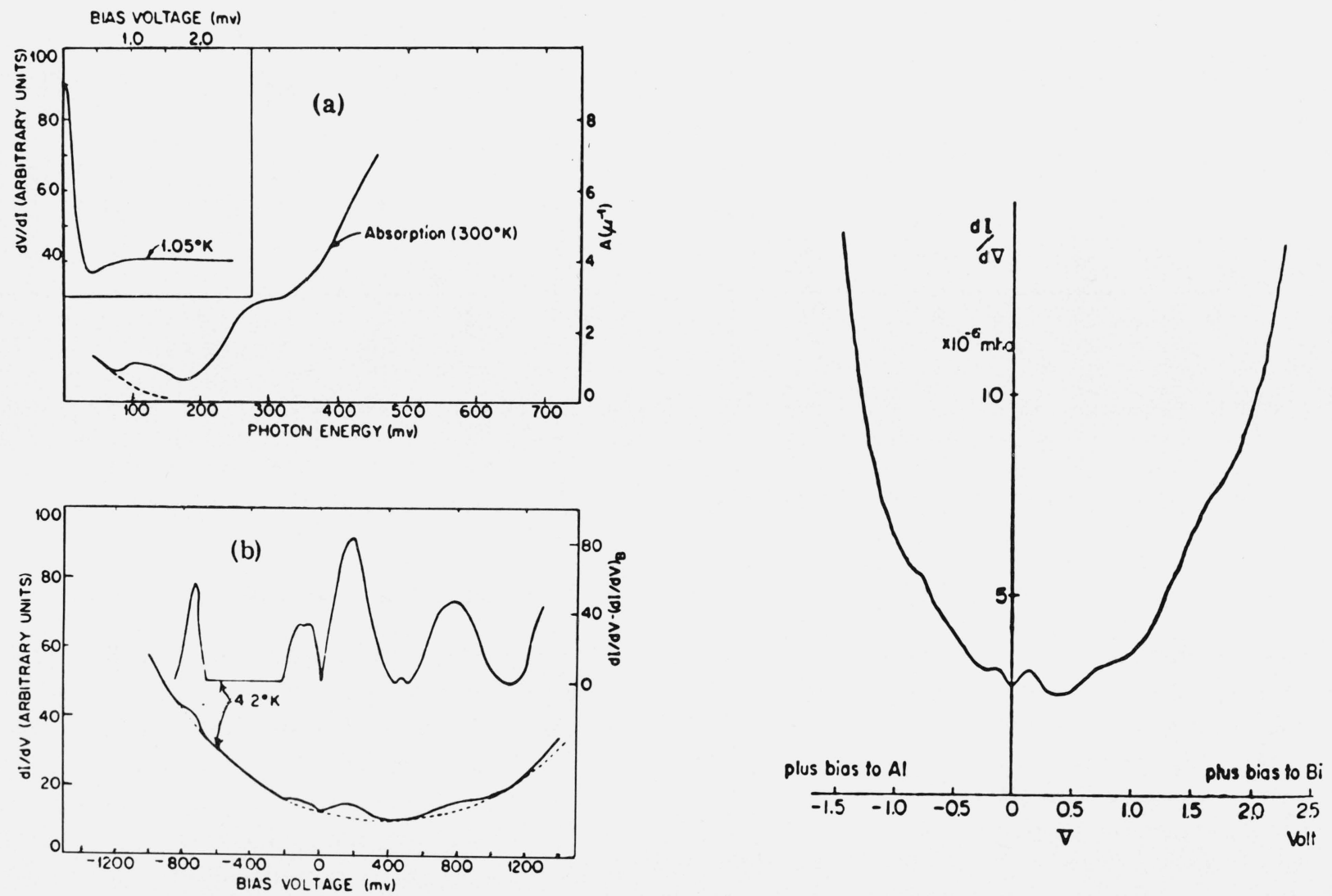

FIgURE 10. Tunneling conductance for Al-I-polycrystalline Bi, as determined by Hauser and Testardi [16] (left figure) and Sawatari and Arai [17] (right figure). (a) Insert: $\mathrm{dV} / \mathrm{dI}$ vs V for an Al-Al $\mathrm{O}_{3}$-Bi junction. Bottom curve, adsorption as a function of energy. The dashed line shows the contribution from free carriers. (b) $\mathrm{d} / \mathrm{dV}$ vs $\mathrm{V}$ (solid line) and background curve (dashed line); curve of ( $\mathrm{d} \mathrm{I} / \mathrm{d} \mathrm{V}$ ) $(\mathrm{dI} / \mathrm{dV})_{b k g}$ vs $\mathrm{V}$. (+ V corresponds to Bi positive.) (c) Conductance-voltage curve in the voltage range $-1.4 \sim+2.3 \mathrm{~V}$ at $77 \mathrm{~K}$. The sample is different from that of $(a)$ and $(b)$. 
The conductance characteristics of M-I-M junctions generally show considerable structure at voltages $<1$ volt, most of which is due to excitation processes in the insulator (oxide). These excitation interactions of the tunneling electron are with organic impurities in the oxide [18], phonons of the oxide [18,19] or phonons of the surfaces of the metal electrodes [19]. In a number of junctions, (Al-I-Ni, Al-I-NiPd alloys [20] and single crystal $\mathrm{Cr}-\mathrm{I}-\mathrm{Pb}[21])$ there are additional effects which are not yet understood. However, it would be unwise to ascribe these to density of states variations without much more detailed experimental work as the barrier properties are, as usual, practically unknown.

\section{Metal-Insulator-Superconductor}

There are two main reasons why tunneling into superconductors has been so successful. First, the important parameter is the normalized density of states, which is the conductance versus voltage with the second electrode superconducting divided by the conductance with it normal. Thus it is not necessary to be able to calculate, or even understand, the normal state characteristic, as long as it is entirely due to tunneling. The barrier parameters, which are exceptionally difficult to determine, can remain as unknowns as long as they do not change when the electrode becomes superconducting. As far as we know, the only change might be in the position of the excitation processes, but these are fortunately weak compared with the superconducting effects. The second advantage of the superconducting measurement is that we can determine essentially bulk properties of the superconductor, whereas all normal state measurements probe the interface and surface properties of the normal electrode. As this technique has been discussed in numerous publications [22], no more detail will be given in this text.

\section{References}

[1] Harrison, W. A., Phys. Rev. 123, 85 (1961).

[2] Davydov, A. S., "Quantum Mechanics," Fizmatgiz, Moscow 1963, English trans. Neo Press, Ann Arbor, 1966.

[3] Duke, C. B., "Tunneling in Solids," Academic Press, New York, 1969, page 55.

[4] Fisher, J. C., and Giaever, I., J. Appl. Phys. 32, 172 (1961).

[5] Conley, J. W., Duke, C. B., Mahan, G. D., and Tiemann, J. J., Phys. Rev. 150, 466 (1966).

[6] Stinrisser, F., Davis, L. C., and Duke, C. B., Phys. Rev. 176, (1967).

[7] Conley, J. W., and Tiemann, J. J., J. Appl. Phys. 38, 2880 (1967)

[8] Chang, L. L., Stiles, P. J., and Esaki, L., J. Appl. Phys. 38, 4440 (1967).

[9] Gray, P. V., Phys. Rev. 140, Al79 (1965).

[10] Esaki, L., Phys. Rev. 109, 603 (1958).

[11] Chynoweth, A. G., Logan, R. A., and Wolff, P. A., Phys. Rev. Letters 5, 548 (1960).

[12] Bernard, W., Goldstein, S., Roth, H., Straub, W. D., and Mulhern, J. E., Jr., Phys. Rev. 166, 785 (1968).

[13] Tsui, D. C., Phys. Rev. Letters 24, 303 (1970).

[14] Esaki, L., and Stiles, P. J., Phys. Rev. Letters 14, 902 (1965); Esaki, L., Chang, L. L., Stiles, P. J., O'Kane, D. F., and Wiser, N., Phys. Rev. 167, 637 (1968).

[15] Giaever, I., Solid State Comm. 5, X (1967) unpublished.

[16] Hauser, J. J., and Testardi, L. R., Phys. Rev. Letters 20, 12 (1968).

[17] Sawatari, Y., Arai, M., Japan J. Appl. Phys. 7, 791 (1968).

[18] Lambe, J., and Jaklevic, R. C., Phys. Rev. 165, 821 (1968).

[19] Rowell, J. M., McMillan, W. L., and Feldmann, W. L., Phys. Rev. 180, 658 (1969).

[20] Rowell, J. M., J. Appl. Phys. 40, 1211 (1969).

[21] Shen, L. Y. L., J. Appl. Phys. (to be published).

[22] McMillan, W. L., and Rowell, J. M., Phys. Rev. Letters 14, 108 (1965); Chapter 11 of "Superconductivity," R. Parks, Editor, Marcel Dekker, New York, 1969.; Rowell, J. M., "Tunneling Phenomena in Solids," S. Lundqvist and E. Burstein, Editors, Plenum Press, New York, 1969.

(Paper 74A4-622) 